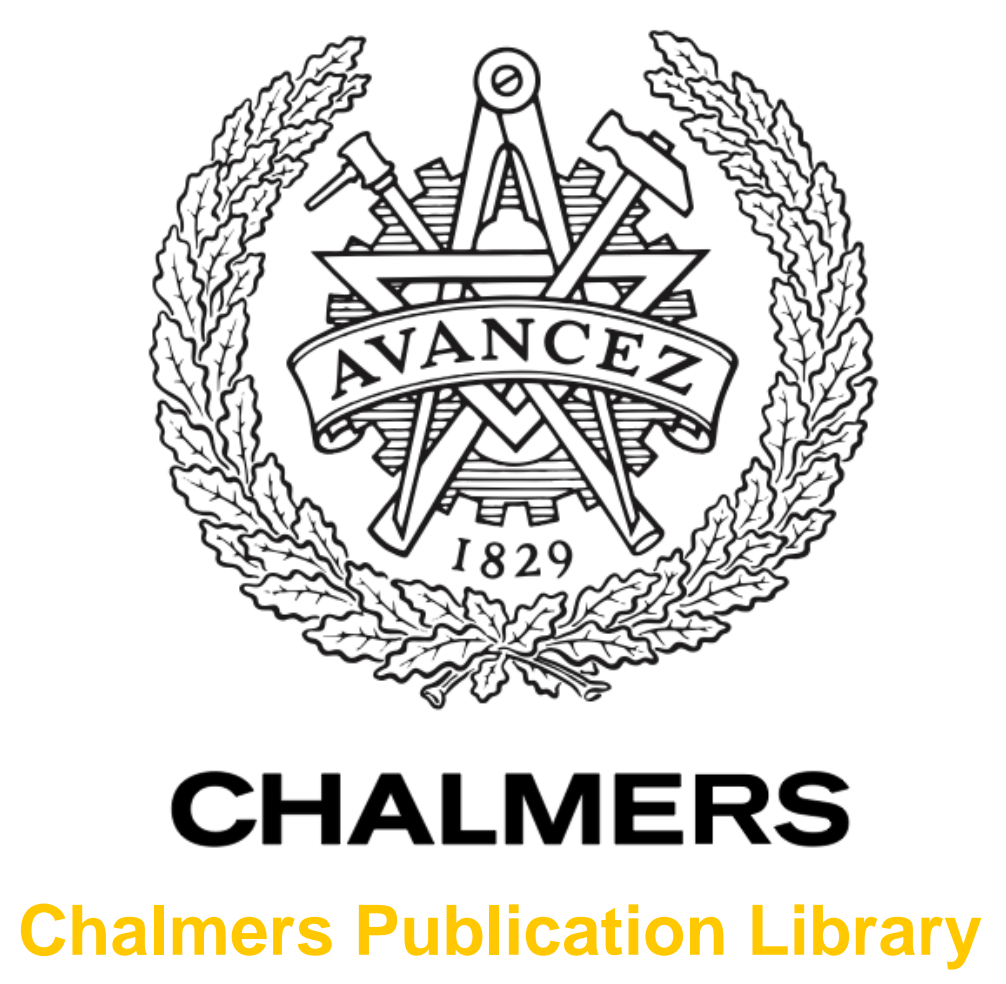

Measurement of the Phase Noise Tracking Capability of a Digital Coherent Receiver

This document has been downloaded from Chalmers Publication Library (CPL). It is the author's version of a work that was accepted for publication in:

Proceedings of European Conference on Optical Communication, ECOC 2011, Geneva, Switzerland, September 18-22, 2011

Citation for the published paper:

Sjödin, M. ; Wymeersch, H. ; Johannisson, P. (2011) "Measurement of the Phase Noise Tracking Capability of a Digital Coherent Receiver". Proceedings of European Conference on Optical Communication, ECOC 2011, Geneva, Switzerland, September 18-22, 2011

Downloaded from: http://publications.lib.chalmers.se/publication/141448

Notice: Changes introduced as a result of publishing processes such as copy-editing and formatting may not be reflected in this document. For a definitive version of this work, please refer to the published source. Please note that access to the published version might require a subscription. 


\title{
Measurement of the Phase Noise Tracking Capability of a Digital Coherent Receiver
}

\author{
Martin Sjödin $^{1}$, Henk Wymeersch ${ }^{2}$, Pontus Johannisson ${ }^{1}$, Magnus Karlsson ${ }^{1}$, and Peter A. Andrekson ${ }^{1}$ \\ 1: Photonics Laboratory, Department of Microtechnology and Nanoscience, Chalmers University of Technology, SE-412 96, Göteborg, Sweden \\ 2: Communication Systems Group, Department of Signals and Systems, Chalmers University of Technology \\ martin.sjodin@chalmers.se
}

\begin{abstract}
We present a method to evaluate the phase noise tracking performance of a digital coherent receiver. Measurements of the phase error variance of QPSK at different symbol rates are presented for two different algorithms.

OCIS codes: (060.1660) Coherent communications; (060.0060) Fiber optics and optical communications.
\end{abstract}

\section{Introduction}

The ability of a coherent receiver to detect the field of an optical signal makes it possible to compensate for linear channel impairments [1], and to use advanced modulation formats such as N-QAM [2]. In addition, the use of digital signal processing (DSP) instead of an optical phase-locked loop facilitates carrier synchronization, a task critical for successful demodulation of the data. A lot of work has been devoted to investigate the tolerable linewidth-symbolperiod product $(\Delta v T)$ of different carrier-recovery schemes [3-5]. These studies are based on numerical simulations in which the laser phase noise is modeled as a Wiener process with variance $\sigma_{p}^{2}=2 \pi \Delta v T$, where $\Delta v$ is the combined linewidth of the signal laser and the local oscillator (LO) laser and $T$ is the symbol period. The phase error variance (PEV) of the estimated phase is often given as a performance measure. However, for several reasons it would be valuable to also be able to experimentally evaluate algorithms for phase noise estimation: Simulations are ideal and it is not always possible to accurately model the stochastic properties of the transmitter and the receiver used in an experiment. In this paper we demonstrate a method to directly measure the PEV of coherently detected signals. The method enables experimental evaluation and comparisons of phase estimation algorithms. We exemplify by using the scheme to measure the PEV of a QPSK signal for two different algorithms: Firstly, we find the optimal block lengths for the block-based Viterbi-Viterbi algorithm [6], and secondly we determine the optimal coefficients of the Wiener filter used in the phase estimation algorithm presented in [3].

\section{Description of the method}

The experimental setup is shown in Fig. 1. The continuous wave (CW) output from a distributed feedback (DFB) laser with a wavelength of $1548 \mathrm{~nm}$ and a linewidth of $900 \mathrm{kHz}$ was split into two paths. In one path, an IQ modulator (IQM) driven by two $2^{15}-1$ PRBS generated a Gray coded QPSK signal at symbol rates from 2.5 Gbaud to 20 Gbaud. A variable attenuator (Att) and an EDFA were used to adjust the OSNR before the receiver and a band-pass filter (BPF) with $1.2 \mathrm{~nm}$ bandwidth suppressed amplified spontaneous emission noise. After the BPF the QPSK signal entered signal input $\mathrm{s}_{\text {sig }}$ of a coherent receiver with polarization diversity, consisting of two optical $90^{\circ}$ hybrids with integrated balanced detectors and electrical amplifiers. The combined $3 \mathrm{~dB}$ bandwidth of the detectors and the amplifiers was more than $25 \mathrm{GHz}$.

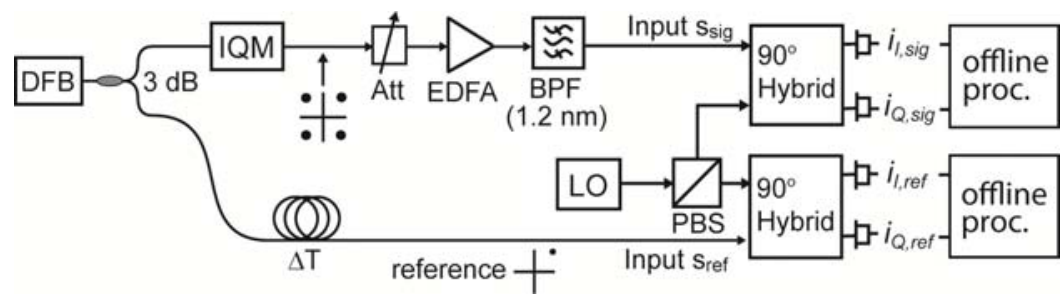

Fig. 1: The experimental setup for measuring the quality of the phase noise tracking of a digital coherent receiver.

The CW light in the second path was used as a reference. It first passed through a piece of fiber and was then coupled to the other signal input $s_{\text {ref }}$ of the coherent receiver. The fiber length was chosen so that its propagation delay $\Delta \mathrm{T}$ ensured a rough alignment in time between the reference and the signal to be measured. To be able to compare their phases, the residual path length mismatch between the two signals was compensated for offline. The LO was an external cavity laser with $500 \mathrm{kHz}$ linewidth. Its output was split into two paths of equal length and coupled into the hybrid LO inputs. 
After photodetection, the complex photocurrents $i_{\text {ref }}$ and $i_{\text {sig }}$ of the reference and the modulated signal were sampled synchronously at $50 \mathrm{Gsample} / \mathrm{s}$ by a real-time sampling oscilloscope with $16 \mathrm{GHz}$ analog bandwidth. $i_{\text {ref }}$ was filtered with a square filter with $250 \mathrm{MHz}$ bandwidth, chosen since it resulted in the smallest PEV in the measurements, and $i_{\text {sig }}$ was filtered with a $5^{\text {th }}$ order Bessel filter with bandwidth equal to a factor of 0.75 times the symbol rate of the QPSK signal. Both $i_{\text {ref }}$ and $i_{\text {sig }}$ were then down-sampled to the QPSK symbol rate. The frequency peak resulting from the beating between the signal laser and the LO laser was obtained from the spectra of the complex photocurrents raised to the fourth power, and the intermediate frequency was removed by a linear phase shift on the data streams.

The carrier recovery was different for $i_{r e f}$ and $i_{s i g}$. For $i_{r e f}$, the phase was estimated and unwrapped directly. For $i_{\text {sig }}$, two different phase estimation algorithms, further described in section 3, were used. The estimated phase noise was compared to the phase noise of the reference and the PEV was measured as a function of the OSNR, the symbol rate and different parameters of the two algorithms. To investigate the performance limits of the scheme, the PEV was first measured with a CW at $48 \mathrm{~dB}$ OSNR as input signal. This resulted in a PEV of $5.0 \cdot 10^{-4} \mathrm{rad}^{2}$.

\section{The carrier recovery schemes}

We study two different feedforward carrier recovery schemes, both using the well-known Viterbi-Viterbi estimator [6]. The phase estimates are given by

$$
\hat{\theta}_{k}=\frac{1}{4} \arg \left\{\sum_{l=m_{k}}^{m_{k}+M-1} i_{l}^{4}\right\}
$$

where $m_{k}$ is the index of the first symbol in the $k$ th block, $i$ is the complex photocurrent and $M$ is the length of a block of samples.

Scheme 1 is based on block processing, in which the samples are segmented into blocks of length $M$ and the phase is considered to be approximately constant within each block. Short block lengths make it possible to track fast phase variations while long blocks are beneficial at low OSNR. In the experiment, we measured the PEV as a function of the OSNR and the block length $M$. We considered block lengths of $4,8, \ldots, 256$ symbols.

Scheme 2 is thoroughly described in [3]. It consists of two stages: in the first stage a symbol-by-symbol estimator (SBSE, $M=1$ ) makes a so-called soft estimation of the phase of each symbol and the phase is unwrapped. A hard phase estimate is obtained in the second stage by applying a Wiener filter to the soft phase estimates. The filter is symmetric and exponentially decaying on both sides of the center tap. The decay rate depends on the filter parameter $r$, which is the ratio between ${\sigma_{p}}^{2}$ and the error variance $\sigma_{n}{ }^{2}$ of the soft phase estimate, $r=\sigma_{p}{ }^{2} / \sigma_{n}{ }^{2}$. In the limit of high OSNR and broad linewidth, the optimal filter has a single tap, while in the limit of low OSNR and narrow linewidth, the filter is long and has slow decay. We determined the optimal value of $r$ for four different QPSK symbol rates at $30 \mathrm{~dB}$ OSNR. With a SBSE as the first phase estimation stage, high OSNR is required for reliable phase estimation without an excessive number of phase cycle slips.

\section{Experimental and numerical results}

Figs. 2a-c show the results for scheme 1. The measured PEV is plotted as a function of the Viterbi-Viterbi block lengths for 5 Gbaud, 10 Gbaud and 20 Gbaud QPSK, respectively. For each case, the optimal block length is decreasing as the OSNR is increased and for long block lengths the phase estimation performance is approximately independent of the OSNR due to the averaging effect. For this reason, the performance improvement with increasing symbol rate ( since $\sigma_{p}^{2} \propto T$ ) is most easily noticed at the longest block lengths, and the PEV increases approximately linearly with the symbol period for $M=256$. For a PEV of $0.010 \mathrm{rad}^{2}$, we measured the OSNR penalty at a BER of $10^{-3}$ to be $0.5 \mathrm{~dB}$ for all symbol rates. This value was also obtained from numerical simulations (not shown here). The smallest measured PEV for scheme 1 is $0.0020 \mathrm{rad}^{2}$, obtained at both 10 and 20 Gbaud (at $30 \mathrm{~dB}$ OSNR) using $M=16$ and $M=32$, respectively.

Fig. 3a shows the measured PEV of the QPSK signal as a function of the filter parameter $r$ in scheme 2 for four different symbol rates, 2.5, 5, 10 and 20 Gbaud. The optimal $r$ value is decreasing with the symbol rate, which is illustrated more clearly in Fig. 3b. This is expected since the tolerance to laser linewidth is increasing linearly with the symbol rate, while $\sigma_{n}^{2}$ increases due to the larger bandwidth. Assuming that $\sigma_{n}{ }^{2}$ increases linearly as a function of the receiver bandwidth, $r$ should be reduced with a factor of four as the symbol rate is doubled, equivalent to a decrease by 0.6 on a 10-logarithmic scale. The expected slope is also plotted in Fig. $3 \mathrm{~b}$ and shows good agreement with the measured data. The best PEV is $0.0017 \mathrm{rad}^{2}$ and is obtained for both 10 and 20 Gbaud. The reason that the phase estimation is not better at 20 Gbaud is that intersymbol interference is becoming significant. Fig. 3c shows the Wiener filters for $r=0.1$ and $r=0.01$. 
There is a discrepancy between the measured $r$ values and those that can be calculated following [3], which are about five times larger. There are several possible reasons, including mismatch between the experimental phase noise and the Wiener model from [3], and possible correlation in the LO-spontaneous beat-noise.
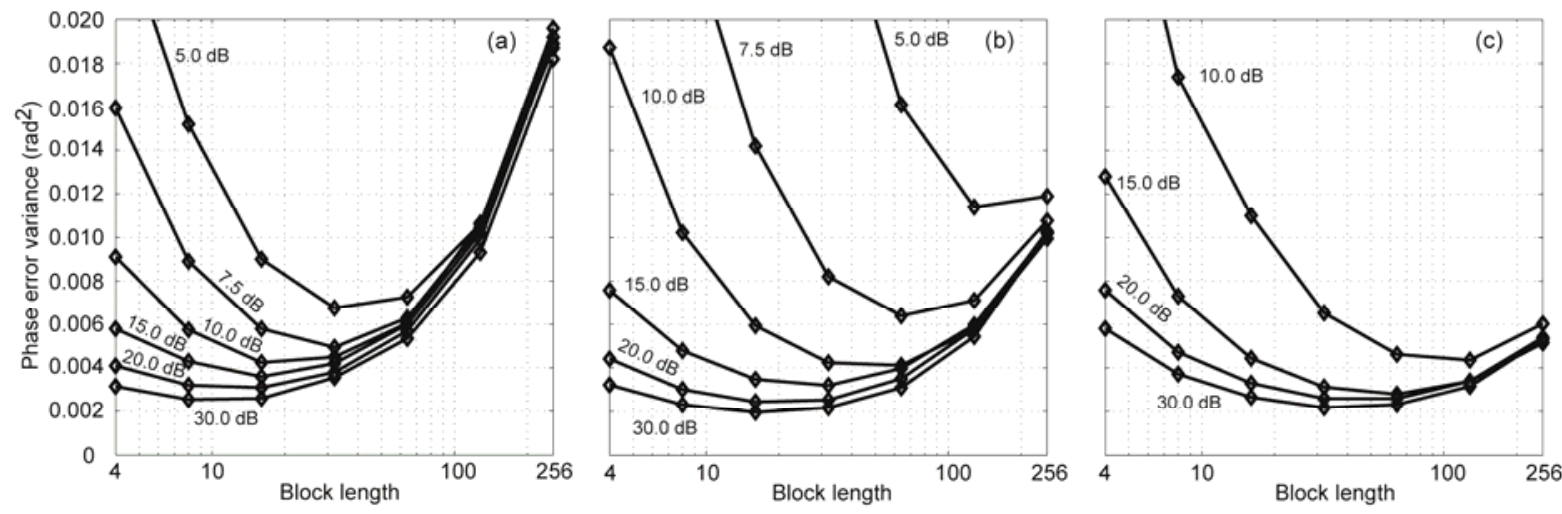

Fig. 2: Measured phase error variance for (a) 5 Gbaud, (b) 10 Gbaud, and (c) 20 Gbaud QPSK as a function of the block length of the Viterbi-Viterbi algorithm and the OSNR before the receiver. The OSNR values are stated for each curve. A PEV of $0.01 \mathrm{rad}^{2}$ gives about $0.5 \mathrm{~dB}$ OSNR penalty at a BER of $10^{-3}$.
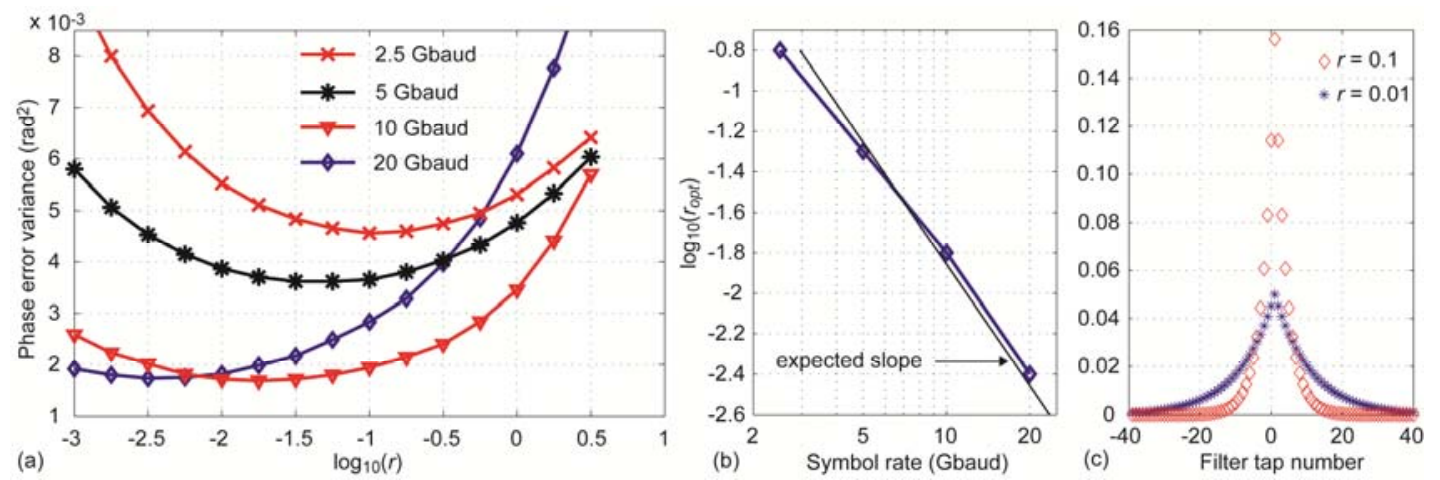

Fig. 3: (a) Measured phase error variance for different symbol rates as a function of $r$. (b) The optimal value of $r$ for different symbol rates together with the expected variation with the symbol rate. (c) The Wiener filters for $r=0.1$ and $r=0.01$.

\section{Conclusions}

We have described a method to measure the phase error variance after phase estimation with a digital coherent receiver, which gives the possibility to evaluate carrier recovery algorithms. The phase of a modulated signal is compared to the phase of a CW at high OSNR. We have used the method to find the optimal block length for the Viterbi-Viterbi algorithm for different symbol rates and OSNRs of a QPSK signal. We also determined the optimal tap values of a Wiener filter used for hard phase estimates in another well-known phase estimation algorithm. The performance of the two phase estimation schemes was similar at high OSNR.

\section{References}

[1] M. G. Taylor, "Coherent detection method using DSP for demodulation of signal and subsequent equalization of propagation impairments," Photonics Technology Letters, vol. 16, no. 2, pp. 674-676, 2004.

[2] J. Hongo et al., "1-Gsymbol/s 64-QAM Coherent Optical Transmission Over 150 km," Photonics Technology Letters, vol. 19, no. 9, pp. 638$640,2007$.

[3] E. Ip et al., "Feedforward Carrier Recovery for Coherent Optical Communications," Journal of Lightwave Technology, vol. 25, no. 9, pp. 2675-2692, 2007.

[4] T. Pfau et al., "Hardware-Efficient Coherent Digital Receiver Concept With Feedforward Carrier Recovery for M-QAM Constellations," Journal of Lightwave Technology, vol. 27, no. 8, pp. 989-999, 2009.

[5] D.-S. Ly-Gagnon et al., "Coherent detection of optical quadrature phase-shift keying signals with carrier phase estimation," Journal of Lightwave Technology, vol. 24, no. 1, pp. 12-21, Jan. 2006.

[6] A. J. Viterbi and A. M. Viterbi, "Nonlinear estimation of PSK-modulated carrier phase with application to burst digital transmission," IEEE Transactions on Information Theory, vol. 29, no. 4, pp. 543-551, 1983. 